\title{
Clinical Applications of Intravascular Ultrasound
}

\author{
Dermot Phelan, Sajjad Matiullah and Faisal Sharif \\ University College Hospital Galway \\ Ireland
}

\section{Introduction}

Intravascular Ultrasound (IVUS) is an invasive grey scale tomographic imaging modality providing cross-sectional images of the vessel wall. The reflected or scattered ultrasound signal received at the transducer is converted to a voltage. This voltage is known as radiofrequency data or backscattered signal. The time delay and amplitude of these emitting pulses provides 256 such backscattered signals or A-scans to produce one image. For IVUS imaging, high ultrasound frequencies typically centred between $25-50 \mathrm{MHz}$ are used. The size of conventional IVUS catheter is 2.9 and $3.5 \mathrm{Fr}$ and has a typical pullback speed of 0.5 $\mathrm{mm} / \mathrm{s}$ and a frame rate of 30 images per second. At $30 \mathrm{MHz}$ the wavelength is $50 \mu \mathrm{m}$, which yields a spatial resolution of $>150 \mu \mathrm{m}$ allowing detail evaluation of the blood vessel wall. IVUS imaging is complementary to coronary angiography and allows the simultaneous assessment of lumen and components of the vessel wall. In the IVUS image the catheter is in the centre of the image surrounded by vessel lumen, the three layers of the vessel and surrounding structures (Figure 1). It can assess plaque geometry including plaque burden and size, luminal area, longitudinal extent of the disease, circumferential extent of the

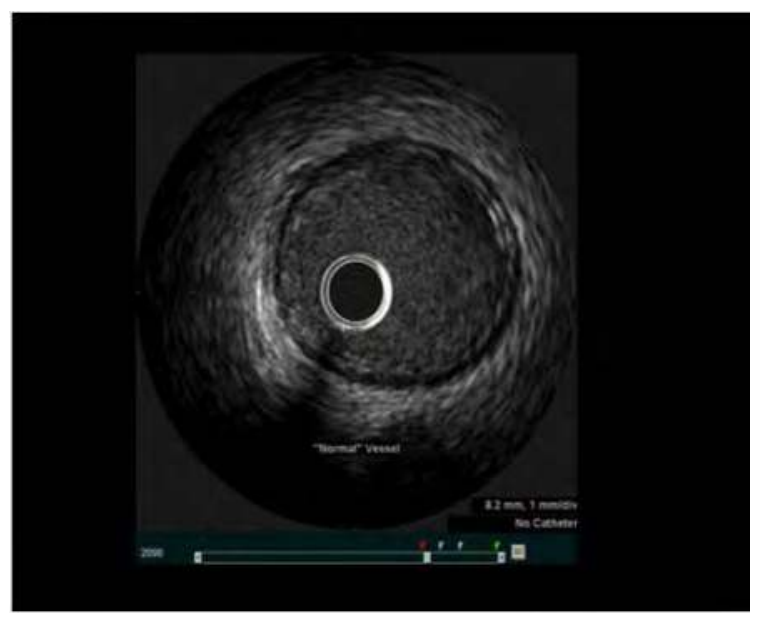

Fig. 1. IVUS of normal coronary artery 
plaque, arterial remodelling and plaque vulnerability. Therefore IVUS provides detailed insight to the anatomy of plaque burden and allows the interventional cardiologist to adapt an optimal strategy for percutaneous coronary intervention (PCI) and subsequently assess the success of this strategy. In this chapter we will describe the potential applications of IVUS for every day use in the catheterization laboratory.

\section{Clinical applications of IVUS}

\subsection{Assessment of vessel size for stent selection and ischemia evaluation}

Standard coronary angiography is intrinsically limited to evaluate three-dimensional anatomical coronary cross sectional area due to planar silhoutte imaging. In addition, the plaque burden, its delineation and constituents cannot be assessed by coronary angiography. With angiography, the severity of stenosis is assessed by minimal lumen diameter at the lesion site, in comparison with an adjacent normal appearing reference. However, it is well documented that atherosclerosis is diffuse in nature and may appear normal in a small calibre coronary artery with concentric plaque (Figure 2)(Grondin, Dyrda et al. 1974; Roberts and Jones 1979). IVUS provides a complete 360 degrees tomographic view that allows accurate lumen measurements. In fact direct comparison of atherosclerotic disease by angiography and IVUS are frequently discrepant often as a result of eccentric plaque.(Figure 2) IVUS studies have clearly demonstrated that there is no correlation between the size of the atheroma and the size of the lumen(Topol and Nissen 1995). This difference can be explained by positive (expansive) remodelling where lumen size is maintained due to plaque accumulation within in the vessel wall and resulting vessel wall expansion. It is claimed that these positive plaques are more unstable and vulnerable to rupture than negative (constrictive) plaques and also responsible for in-stent restenosis (ISR) following coronary intervention.(Schoenhagen, Ziada et al. 2000).
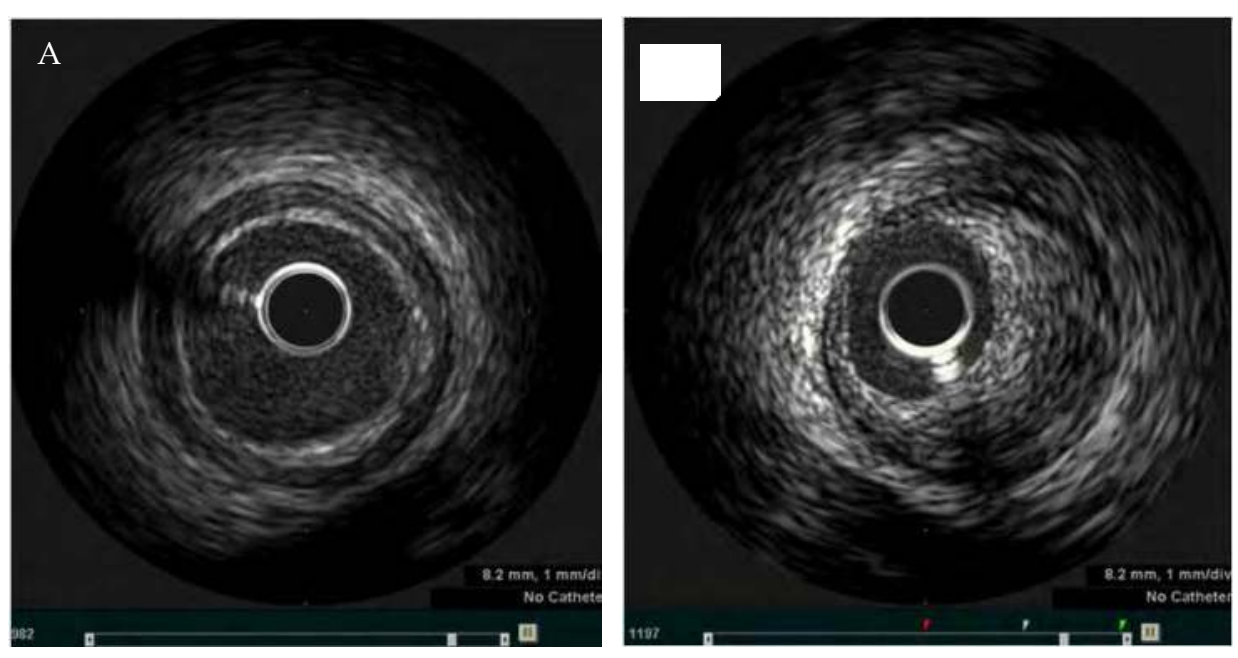

Fig. 2. IVUS images of A) concentric plaque and B) eccentric plaque 
In addition to accurate stent sizing, IVUS can be used to evaluate lesion lumen area to predict the presence or absence of myocardial ischemia and/or significant coronary stenosis. There was strong correlation between lesion lumen area of $<4.0 \mathrm{~mm} 2$ on IVUS and positive stress myocardial perfusion SPECT in seventy native coronary lesions (Nishioka, Amanullah et al. 1999). In another study, IVUS guided deferral of coronary intervention of 248 lesions with luminal area of $>4 \mathrm{~mm}^{2}$ resulted in clinical rates of $4.4 \%$ and target lesion revascularisation (TLR) of $2.8 \%$ at 12 month follow up. (Abizaid, Mintz et al. 1998).

\subsection{Assessment of restenosis}

Following coronary intervention, the formation of neointimal hyperplasia is mainly responsible for ISR (Figure 3). There is strong correlation with between late lumen loss and the degree of in-stent neointimal growth $(\mathrm{r}=0.98)$ (de Jaegere, Mudra et al. 1998; Hoffmann, Mintz et al. 1998). Although drug eluting stents (DES) has significantly reduced the incidence of neointimal proliferation, the rate of ISR still remains around 10-15\%.

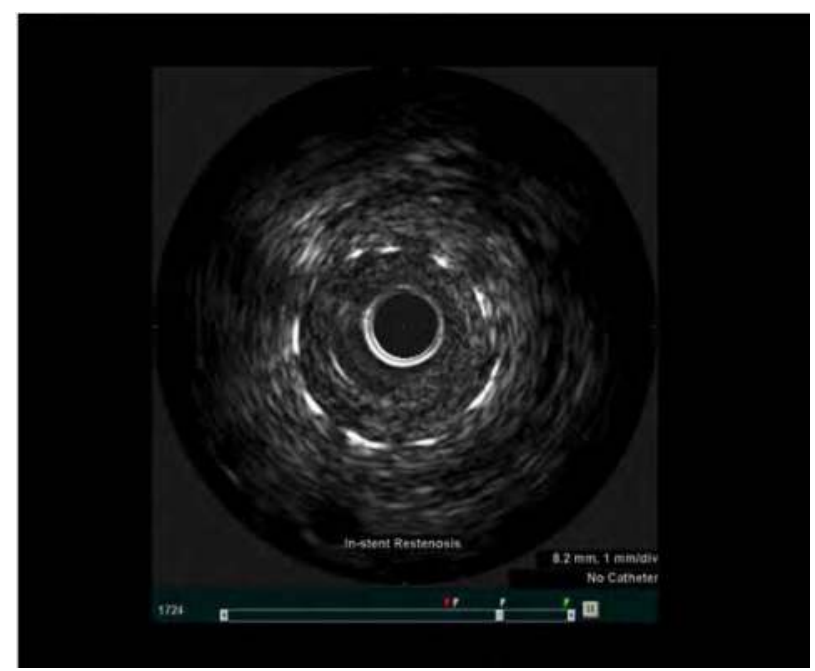

Fig. 3. IVUS image of in-stent restenosis

One of the main factors for ISR is stent underexpansion (Figure 4). IVUS can detect vessel stent subtleties not apparent by coronary angiography. In addition to assessing plaque geometry, IVUS can be used to achieve complete stent apposition and adequate geometric expansion within the stented segment. IVUS studies have demonstrated that incomplete stent and vessel wall apposition, residual stenosis and irregular eccentric lumen in the stented segment was present in almost $88 \%$ of the patients despite achieving an optimal angiographic result (Nakamura, Colombo et al. 1994) . Clinical trials have shown that patients who have their coronary intervention guided by IVUS have larger post procedure stent areas and significant reductions in TLR as compared to angiography-guided PCI alone (de Jaegere, Mudra et al. 1998; Schiele, Meneveau et al. 1998; Fitzgerald, Oshima et al. 2000; Sonoda, Morino et al. 2004; Hong, Mintz et al. 2006) In addition to detecting stent 
underexpansion, IVUS can also assist in achieving optimal stent expansion, exclude stent edge dissections and plaque protrusion (Hong, Jeong et al. 2008).

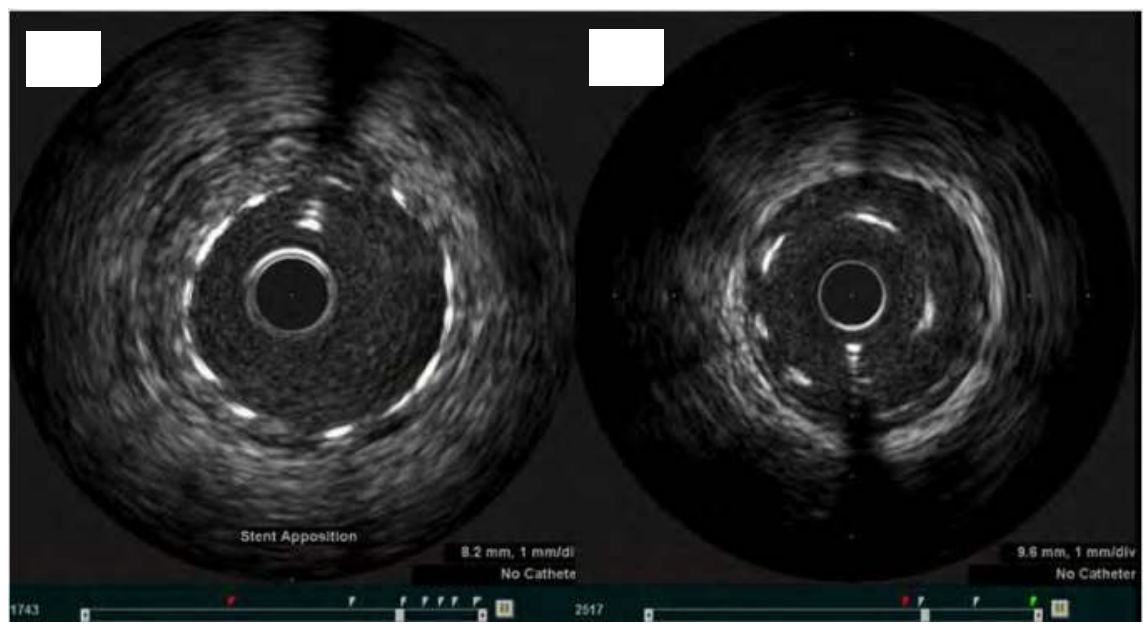

Fig. 4. IVUS demonstrating A) stent well apposed to vessel wall and B) stent mal-apposition

\subsection{Guidance for left main stem intervention}

Coronary interventions of unprotected left main coronary artery (UPLM) with bare metal stents in the 1990s were associated with high rates of revascularization (25\% to 30\%) due to restenosis (Black, Cortina et al. 2001; Park, Hong et al. 2001; Takagi, Stankovic et al. 2002). Although the use of DES for UPLM PCI have significantly reduced the rate of revascularization as compared with bare metal stents (BMS), the long term outcomes remain less favourable. Most of the poor outcomes following UPLM PCI relates to distal bifurcation intervention, which is a technically complex procedure. The current American College of Cardiology (ACC)/American Heart association (AHA) practice guidelines for PCI categorize UPLM stenting as a class IIb indication or IIa indication in selected patients without co-existing multivessel disease. At present there is a paucity of randomized control trial data comparing Coronary artery bypass grafting (CABG) to PCI in patients with UPLM. The best available evidence comes from the SYNTAX trial (SYNergy between Percutaneous Coronary Intervention with TAXus and Cardiac Surgery), which randomly assigned 1800 patients with either UPLM $(n=705)$ or multivessel CAD not involving the left main stem $(n=1095)$ (Ong, Serruys et al. 2006) . The major adverse cardiac or cerebrovascular events were not significantly different between the CABG and PCI groups (13.7 vs 15.8\%). However, the rate of revascularization was significantly higher in those treated with PCI (11.8 vs $6.5 \%$ ). One of the main limitations of the SYNTAX trial was felt to be the lack of use of IVUS for UPLM in the PCI group.

While the European Society of Cardiology guidelines give IVUS-guided stenting of the UPLM a Class IIb recommendation we feel IVUS interrogation of the UPLM pre and post intervention should be used in all cases. Not least because IVUS interrogation has been demonstrated to, frequently prove angiographic assessment of the UPLM as inaccurate. This obviously may have significant impact on patient management resulting in either erroneous 
treatment of a lesion which may appear severe angiographically but in fact has minimal plaque burden or failure to treat lesions which are mis-labelled as mild-to-moderate (Mintz and Maehara 2009).

Moreover, IVUS is an ideal method for confirming the presence of significant left main disease and also for guiding selection of stent size, assessing the presence and extent of calcification and especially evaluating the distal left main vessel and its branches. IVUS assessment pre and post left main intervention is very important to evaluate larger lumen area of the ostial and midshaft left main and adequate post dilatation post stenting. In comparison to native coronary vessels, a minimal luminal area of $<6.0 \mathrm{~mm} 2$ is a commonly used threshold for significant left main disease (Sano, Mintz et al. 2007). While there are no randomized trials to inform practice in this area, registry data has shown a trend toward reduced mortality in IVUS guided UPLM PCI.(Park, Kim et al. 2009)

\subsection{Guidance for Chronic Total Occlusion (CTO) intervention}

CTOs are the most complex lesions that are considered for percutaneous coronary revascularization. PCI of CTO results in symptomatic improvement, improved left ventricle function and reduction in adverse remodelling. In addition recanalization of CTO leads to long-term survival benefit and avoidance of bypass surgery (Melchior, Doriot et al. 1987; Ivanhoe, Weintraub et al. 1992; Chung, Nakamura et al. 2003; Cheng, Selvanayagam et al. 2008). The number of CTO interventions has risen gradually due to better operator experience, technical improvements, and newer procedural techniques (these include contra-lateral coronary injection, "parallel" wire techniques, subintimal tracking and re-entry (STAR), retrograde approach with control antegrade retrograde tracking (CART), reverse CART). Despite these advances, the success rate of CTO interventions remains low $(<60 \%)$ (Di Mario, Werner et al. 2007), largely due to difficulty crossing the occlusion with the guidewire and entering the true distal lumen beyond the occlusion (Safian, McCabe et al. 1988; Kinoshita, Katoh et al. 1995). In most instances of failed CTO intervention, the guidewire enters the false lumen (subintimal space) at the site of occlusion, often making it impossible to re-enter the true lumen (Figure 5). CTO interventions can be performed via an antegrade approach or retrograde approach using septal collaterals (Surmely, Tsuchikane et al. 2006).

IVUS studies provide insights into the anatomy of the CTO lesion. In one report IVUS demonstrated presence of calcium mostly across the side branch take-off, especially in abrupt-origin CTOs (Fujii, Mintz et al. 2006). This anatomical variance can explain the preferential entry of the guidewire into the side branch at the point of occlusion. The use of IVUS for CTO interventions can be extremely useful especially in ensuring that the guidewire is positioned within the coronary lumen (true or false), and also helps to identify the optimal entry point within the CTO cap(Ochiai, Ogata et al. 2006). IVUS can help to avoid subintimal stenting during CTO intervention as this has been reported to result in stent thrombosis and stent mal-apposition due to the formation of multiple aneurysms (Erlich, Strauss et al. 2006; Tsujita, Maehara et al. 2009). IVUS imaging for CTO can also detect vessel wall haematoma, dissection and small perforations that are not detected by routine coronary angiography. Further advances in IVUS technology especially the development of 'forward facing IVUS' will significantly improve our anatomical understanding of the chronically occluded lumen. Although IVUS guided intervention of chronic occlusions may enhance procedural outcome, the current use of this technology for CTO intervention is limited only to experienced operators. 


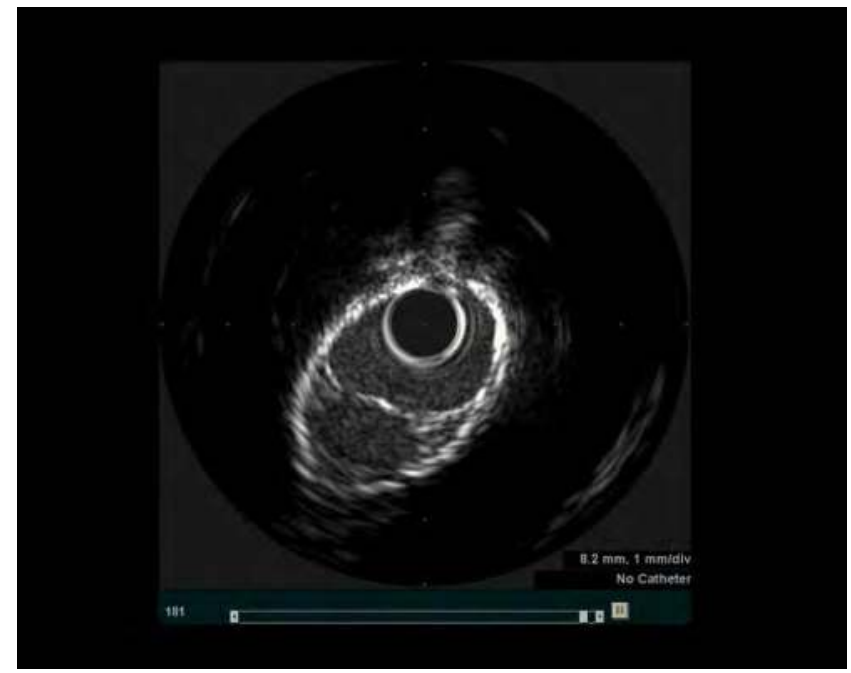

Fig. 5. IVUS image of false lumen

\subsection{Guidance for bifurcation lesion intervention}

PCI of bifurcation lesions have been associated with lower procedural success rate and worse clinical outcome than when used to treated non-bifurcation lesions. This is largely related to the complexity of the bifurcation lesions, significant anatomical variation of the bifurcation lesion, and lack of standard guidelines for the treatment of bifurcation lesions. The current therapeutic strategies for bifurcation lesions have mainly stemmed from personal clinical experience of the operators. (Suzuki, Angiolillo et al. 2007). In recent years there has been better understanding of bifurcation lesions especially with use of DES, acceptance of provisional stenting (acceptance of a suboptimal result in a small side branch $\mathrm{SB})$, specific treatment of bifurcation lesions with a two-stent strategy and increasing use of final kissing balloon. The use of IVUS for bifurcation lesions can provide valuable information especially in anatomical evaluation of plaque burden, plaque location, angle assessment, lumen size of main branch [MB] and [SB]. In addition post PCI, IVUS can assist in evaluation of plaque shift, change in carina angle, dissection, and above all optimal stent deployment (Costa, Mintz et al. 2005). We feel that IVUS guided optimization of the bifurcation lesion post intervention, especially the ostium of a large side branch will enhance long term outcome of these technically challenging subset of lesions.

\subsection{Vulnerable plaque assessment}

The composition of atherosclerotic plaque is heterogeneous by nature and contains 1) fibrocellular components (extracellular matrix and smooth muscle cells), 2) lipid-cellular components (crystalline cholesterol and cholesterol esters mixed with macrophages), 3) thrombotic components (platelets and fibrin) and 4) calcium (Figure 6) (Fuster, Badimon et al. 1992; Fuster, Badimon et al. 1992; Stary 2000; Virmani, Kolodgie et al. 2000). Vulnerable plaques that result in rupture have been now well described as thin cap fibroatheroma (TCFA). 


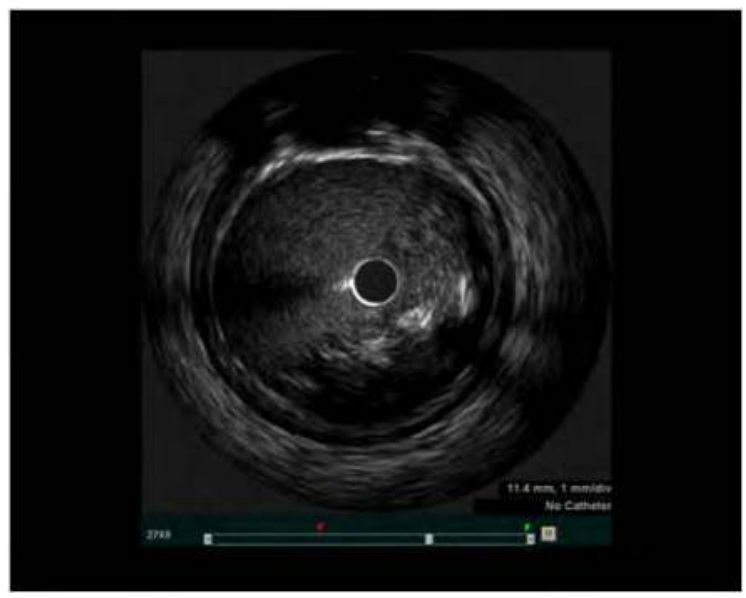

Fig. 6. IVUS image of atherosclerotic plaque

Ultrasound provides some information about the morphological features of atherosclerotic plaque. The American College of Cardiology Clinical Expert Consensus Document for IVUS imaging has described that a vulnerable plaque can appear as an 'echolucent' plaque (Mintz, Nissen et al. 2001). Frequently these echolucent plaques have a prominent echogenic border at the lumen-intima interface. This may correspond to the TCFA. However, it should be emphasized that the fibrous cap of TCFA is $<65 \mu \mathrm{m}$ and therefore could not be detected using this modality. Imaging IVUS provides limited insight into the chemical composition of the plaque and is dependent on simple interpretation of acoustic reflections. Lipid-laden echolucent plaques can be detected with a sensitivity of between $78 \%$ and $95 \%$ and specificity of 30\% (Mallery, Tobis et al. 1990; Potkin, Bartorelli et al. 1990). Furthermore, reduced echogenicity may also be observed in large necrotic zones, intramural hemorrhage or a thrombus.

Echodense plaques have an intermediate echogenicity between echolucent and highly echogenic calcified plaques. The echodensity of plaques correlate well with plaque fibrosis in histological studies. The identification of echolucent plaques is subjective and no prospective clinical trials are available. In an IVUS based study, echolucent plaque was identified in 31 patients from a total of 144 patients. Of these 31 patients, 23 patients $(74 \%)$ presented with unstable angina. Plaque rupture was confirmed by injecting contrast with subsequent filling of the plaque cavity on IVUS (Ge, Haude et al. 1995). In another study, IVUS images of 114 coronary lesions with $<50 \%$ stenosis were recorded and followed up for two years.(Yamagishi, Terashima et al. 2000). The patients who developed Acute coronary syndrome during the follow up were identified and events correlated with large echolucent zones in the eccentric plaques at the time of their first IVUS study. These plaques had histological features similar to vulnerable plaques and were associated with increased risk of instability. In addition, IVUS can also identify vulnerable plaques at high risk of rupture in vessels with adaptive remodelling (von Birgelen, Klinkhart et al. 2001).

IVUS has excellent blood penetration and in the absence of calcium is able to visualize and calculate plaque area, volume and eccentricity. The use of IVUS to identify TCFA and lipid pool is at present limited. It suffers from signal attenuation and geometric effects that result in different backscatter signal properties from similar tissues due to differences in tissue 
orientation and position relative to the imaging transducer. Ultra- high frequency catheters can be employed to achieve high resolution and visualize subluminal fibrous cap, while low-resolution components can be used for better penetration of the plaque to assess plaque components.

The conventional IVUS image undergoes considerable processing such as envelope detection, time-gain compensation, and logarithmic compression to create real time imaging. However, this processing significantly reduces the ability to characterize the imaged tissue, the echogenicity of the imaged tissue is difficult to repeat and interpret quantitatively. The reflected unprocessed ultrasound signal in the form of A-scan or individual backscattered signals (256 A scans for an image) is called the radiofrequency data and it is the analysis of these individual A-scans that holds potential for tissue characterization. This imaging modality is known as IVUS virtual histology (IVUS -VH) or IVUS radiofrequency (IVUS-RF).

Ex-vivo studies have demonstrated that raw backscattered ultrasound signal allows a more detailed analysis of the vessel components with scope to identify different tissue morphology (Moore, Spencer et al. 1998). IVUS-VH uses spectral analysis of radiofrequency data to construct tissue maps that classify plaque into four major components (fibrous [labeled green], fibrolipidic [labeled greenish-yellow], necrotic core [labeled red] and calcium [labeled white]) which were correlated with a specific spectrum of the radiofrequency signal and assigned color codes (Nair, Kuban et al. 2001). In a clinical study, IVUS-VH was used to investigate the presence of IVUS-derived thin cap fibroatheroma (IDTCFA) in non-culprit, non-obstructive $(<50 \%)$ lesions in 55 patients who presented with acute coronary syndrome (Rodriguez-Granillo, Garcia-Garcia et al. 2005). The axial resolution of the IVUS-VH is between 100 to $150 \mu \mathrm{m}$ and therefore in this study the authors assumed that the absence of visible atheroma tissue overlying a necrotic core would suggest a cap thickness of below 100 to $150 \mu \mathrm{m}$ and used the absence of such tissue to define a thin fibrous cap. In this study, IDTCFA was defined and identified as a lesion with a necrotic core $\geq 10 \%$ without evident overlying fibrous tissue and percent atheroma volume (PAV) $\geq 40 \%$. In this study significantly higher prevalence of IDTCFA was observed in patients with Acute coronary syndrome in comparison with controls [(3.0 IQR 0.0 to 5.0 vs. 1.0 IQR 1.0 to 2.8) $\mathrm{p}=0.018$ ]. The large multicenter PROSPECT trial (700 patients with Acute coronary syndrome) looked at long-term outcomes of non-culprit lesions (based on IVUS-VH) at the time of Percutaneous coronary intervention of the culprit lesions. The investigators reported that only 11 percent of the patients had high event rate (i.e. 17\%) in association with thincapped fibroatheromas with minimal luminal area (MLA) $\leq 4 \mathrm{~mm}^{2}$ and plaque burden $\geq 70 \%$. Although high-risk focal sites can be detected with IVUS-VH, the predictive power (significantly higher numbers of vulnerable plaques than clinical events) of vulnerable plaque to cause a clinical event remains low.

IVUS-RF data acquisition and real time processing with three dimensional imaging and spectral analysis is a potential tool to assess vulnerable plaque in vivo. This imaging tool provides detailed volumetric assessment of the histological components of the plaque in vivo and therefore may represent a unique technique to identify the vulnerable plaque in future.

\section{Conclusion}

Over the past decade there has been a significant technological advance in cardiovascular imaging that has changed the way we assess coronary atherosclerosis and approach 
coronary intervention. IVUS is a validated clinical tool that allows precise evaluation of angiographically stenotic lesions helping guide the cardiologist's approach to revascualisation and assess complications and sub-optimal results post-procedure. In addition it provides important information regarding non-critical but vulnerable plaque which is not appreciable by traditional coronary angiography. In an era of more complex and ambitious coronary intervention IVUS is a vitally important addition to the interventionalist's armamentarium. In this chapter we have briefly described the various potential uses of IVUS and their clinical application.

\section{References}

Abizaid, A., G. S. Mintz, et al. (1998). "Clinical, intravascular ultrasound, and quantitative angiographic determinants of the coronary flow reserve before and after percutaneous transluminal coronary angioplasty." Am J Cardiol 82(4): 423-8.

Black, A., R. Cortina, et al. (2001). "Unprotected left main coronary artery stenting: correlates of midterm survival and impact of patient selection." J Am Coll Cardiol 37(3): 832-8.

Cheng, A. S., J. B. Selvanayagam, et al. (2008). "Percutaneous treatment of chronic total coronary occlusions improves regional hyperemic myocardial blood flow and contractility: insights from quantitative cardiovascular magnetic resonance imaging." JACC Cardiovasc Interv 1(1): 44-53.

Chung, C. M., S. Nakamura, et al. (2003). "Effect of recanalization of chronic total occlusions on global and regional left ventricular function in patients with or without previous myocardial infarction." Catheter Cardiovasc Interv 60(3): 368-74.

Costa, R. A., G. S. Mintz, et al. (2005). "Bifurcation coronary lesions treated with the "crush" technique: an intravascular ultrasound analysis." J Am Coll Cardiol 46(4): 599-605.

de Jaegere, P., H. Mudra, et al. (1998). "Intravascular ultrasound-guided optimized stent deployment. Immediate and 6 months clinical and angiographic results from the Multicenter Ultrasound Stenting in Coronaries Study (MUSIC Study)." Eur Heart J 19(8): 1214-23.

Di Mario, C., G. S. Werner, et al. (2007). "European perspective in the recanalisation of Chronic Total Occlusions (CTO): consensus document from the EuroCTO Club." EuroIntervention 3(1): 30-43.

Erlich, I., B. H. Strauss, et al. (2006). "Stent thrombosis following the STAR technique in a complex RCA chronic total occlusion." Catheter Cardiovasc Interv 68(5): 708-12.

Fitzgerald, P. J., A. Oshima, et al. (2000). "Final results of the Can Routine Ultrasound Influence Stent Expansion (CRUISE) study." Circulation 102(5): 523-30.

Fujii, K., G. S. Mintz, et al. (2006). "Intravascular ultrasound profile analysis of ruptured coronary plaques." Am J Cardiol 98(4): 429-35.

Fuster, V., L. Badimon, et al. (1992). "The pathogenesis of coronary artery disease and the acute coronary syndromes (1)." N Engl J Med 326(4): 242-50.

Fuster, V., L. Badimon, et al. (1992). "The pathogenesis of coronary artery disease and the acute coronary syndromes (2)." N Engl J Med 326(5): 310-8.

Ge, J., M. Haude, et al. (1995). "Silent healing of spontaneous plaque disruption demonstrated by intracoronary ultrasound." Eur Heart J 16(8): 1149-51.

Grondin, C. M., I. Dyrda, et al. (1974). "Discrepancies between cineangiographic and postmortem findings in patients with coronary artery disease and recent myocardial revascularization." Circulation 49(4): 703-8. 
Hoffmann, R., G. S. Mintz, et al. (1998). "Intimal hyperplasia thickness at follow-up is independent of stent size: a serial intravascular ultrasound study." Am J Cardiol 82(10): 1168-72.

Hong, M. K., G. S. Mintz, et al. (2006). "Intravascular ultrasound predictors of angiographic restenosis after sirolimus-eluting stent implantation." Eur Heart J 27(11): 1305-10.

Hong, Y. J., M. H. Jeong, et al. (2008). "Plaque prolapse after stent implantation in patients with acute myocardial infarction: an intravascular ultrasound analysis." JACC Cardiovasc Imaging 1(4): 489-97.

Ivanhoe, R. J., W. S. Weintraub, et al. (1992). "Percutaneous transluminal coronary angioplasty of chronic total occlusions. Primary success, restenosis, and long-term clinical follow-up." Circulation 85(1): 106-15.

Kinoshita, I., O. Katoh, et al. (1995). "Coronary angioplasty of chronic total occlusions with bridging collateral vessels: immediate and follow-up outcome from a large singlecenter experience." J Am Coll Cardiol 26(2): 409-15.

Mallery, J. A., J. M. Tobis, et al. (1990). "Assessment of normal and atherosclerotic arterial wall thickness with an intravascular ultrasound imaging catheter." Am Heart J 119(6): 1392-400.

Melchior, J. P., P. A. Doriot, et al. (1987). "Improvement of left ventricular contraction and relaxation synchronism after recanalization of chronic total coronary occlusion by angioplasty." J Am Coll Cardiol 9(4): 763-8.

Mintz, G. S. and A. Maehara (2009). "Serial intravascular ultrasound assessment of atherosclerosis progression and regression. State-of-the-art and limitations." Circ J 73(9): 1557-60.

Mintz, G. S., S. E. Nissen, et al. (2001). "American College of Cardiology Clinical Expert Consensus Document on Standards for Acquisition, Measurement and Reporting of Intravascular Ultrasound Studies (IVUS). A report of the American College of Cardiology Task Force on Clinical Expert Consensus Documents." J Am Coll Cardiol 37(5): 1478-92.

Moore, M. P., T. Spencer, et al. (1998). "Characterisation of coronary atherosclerotic morphology by spectral analysis of radiofrequency signal: in vitro intravascular ultrasound study with histological and radiological validation." Heart 79(5): 459-67.

Nair, A., B. D. Kuban, et al. (2001). "Assessing spectral algorithms to predict atherosclerotic plaque composition with normalized and raw intravascular ultrasound data." Ultrasound Med Biol 27(10): 1319-31.

Nakamura, S., A. Colombo, et al. (1994). "Intracoronary ultrasound observations during stent implantation." Circulation 89(5): 2026-34.

Nishioka, T., A. M. Amanullah, et al. (1999). "Clinical validation of intravascular ultrasound imaging for assessment of coronary stenosis severity: comparison with stress myocardial perfusion imaging." J Am Coll Cardiol 33(7): 1870-8.

Ochiai, M., N. Ogata, et al. (2006). "Intravascular ultrasound guided wiring for chronic total occlusions." Indian Heart J 58(1): 15-20.

Ong, A. T., P. W. Serruys, et al. (2006). "The SYNergy between percutaneous coronary intervention with TAXus and cardiac surgery (SYNTAX) study: design, rationale, and run-in phase." Am Heart J 151(6): 1194-204. 
Park, S. J., M. K. Hong, et al. (2001). "Elective stenting of unprotected left main coronary artery stenosis: effect of debulking before stenting and intravascular ultrasound guidance." J Am Coll Cardiol 38(4): 1054-60.

Park, S. J., Y. H. Kim, et al. (2009). "Impact of intravascular ultrasound guidance on longterm mortality in stenting for unprotected left main coronary artery stenosis." Circ Cardiovasc Interv 2(3): 167-77.

Potkin, B. N., A. L. Bartorelli, et al. (1990). "Coronary artery imaging with intravascular high-frequency ultrasound." Circulation 81(5): 1575-85.

Roberts, W. C. and A. A. Jones (1979). "Quantitation of coronary arterial narrowing at necropsy in sudden coronary death: analysis of 31 patients and comparison with 25 control subjects." Am J Cardiol 44(1): 39-45.

Rodriguez-Granillo, G. A., H. M. Garcia-Garcia, et al. (2005). "In vivo intravascular ultrasound-derived thin-cap fibroatheroma detection using ultrasound radiofrequency data analysis." J Am Coll Cardiol 46(11): 2038-42.

Safian, R. D., C. H. McCabe, et al. (1988). "Initial success and long-term follow-up of percutaneous transluminal coronary angioplasty in chronic total occlusions versus conventional stenoses." Am J Cardiol 61(14): 23G-28G.

Sano, K., G. S. Mintz, et al. (2007). "Assessing intermediate left main coronary lesions using intravascular ultrasound." Am Heart J 154(5): 983-8.

Schiele, F., N. Meneveau, et al. (1998). "Impact of intravascular ultrasound guidance in stent deployment on 6-month restenosis rate: a multicenter, randomized study comparing two strategies--with and without intravascular ultrasound guidance. RESIST Study Group. REStenosis after Ivus guided STenting." J Am Coll Cardiol 32(2): 320-8.

Schoenhagen, P., K. M. Ziada, et al. (2000). "Extent and direction of arterial remodeling in stable versus unstable coronary syndromes : an intravascular ultrasound study." Circulation 101(6): 598-603.

Sonoda, S., Y. Morino, et al. (2004). "Impact of final stent dimensions on long-term results following sirolimus-eluting stent implantation: serial intravascular ultrasound analysis from the sirius trial." J Am Coll Cardiol 43(11): 1959-63.

Stary, H. C. (2000). "Natural history and histological classification of atherosclerotic lesions: an update." Arterioscler Thromb Vasc Biol 20(5): 1177-8.

Surmely, J. F., E. Tsuchikane, et al. (2006). "New concept for CTO recanalization using controlled antegrade and retrograde subintimal tracking: the CART technique." $J$ Invasive Cardiol 18(7): 334-8.

Suzuki, N., D. J. Angiolillo, et al. (2007). "Percutaneous coronary intervention of bifurcation coronary disease." Minerva Cardioangiol 55(1): 57-71.

Takagi, T., G. Stankovic, et al. (2002). "Results and long-term predictors of adverse clinical events after elective percutaneous interventions on unprotected left main coronary artery." Circulation 106(6): 698-702.

Topol, E. J. and S. E. Nissen (1995). "Our preoccupation with coronary luminology. The dissociation between clinical and angiographic findings in ischemic heart disease." Circulation 92(8): 2333-42.

Tsujita, K., A. Maehara, et al. (2009). "Cross-sectional and longitudinal positive remodeling after subintimal drug-eluting stent implantation: multiple late coronary aneurysms, 
stent fractures, and a newly formed stent gap between previously overlapped stents." JACC Cardiovasc Interv 2(2): 156-8.

Virmani, R., F. D. Kolodgie, et al. (2000). "Lessons from sudden coronary death: a comprehensive morphological classification scheme for atherosclerotic lesions." Arterioscler Thromb Vasc Biol 20(5): 1262-75.

Von Birgelen, C., W. Klinkhart, et al. (2001). "Plaque distribution and vascular remodeling of ruptured and nonruptured coronary plaques in the same vessel: an intravascular ultrasound study in vivo." J Am Coll Cardiol 37(7): 1864-70.

Yamagishi, M., M. Terashima, et al. (2000). "Morphology of vulnerable coronary plaque: insights from follow-up of patients examined by intravascular ultrasound before an acute coronary syndrome." J Am Coll Cardiol 35(1): 106-11.

Wu, X., Maehara A, et al. (2010). "Virtual histology intravascular ultrasound analysis of nonculprit attenuated plaques detected by grayscale intravascular ultrasound in patients with acute coronary syndromes." J Am Coll Cardiol 105(1):48-53.

Gregg W. Stone., Akiko Maehara, et al. (2011). " A Prospective Natural-History Study of Coronary Atherosclerosis". N Engl J Med 364:226-35. 


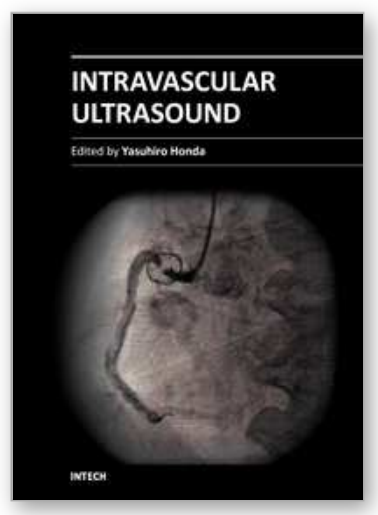

\author{
Intravascular Ultrasound \\ Edited by Dr. Yasuhiro Honda
}

ISBN 978-953-307-900-4

Hard cover, 207 pages

Publisher InTech

Published online 01, February, 2012

Published in print edition February, 2012

Intravascular ultrasound (IVUS) is a cardiovascular imaging technology using a specially designed catheter with a miniaturized ultrasound probe for the assessment of vascular anatomy with detailed visualization of arterial layers. Over the past two decades, this technology has developed into an indispensable tool for research and clinical practice in cardiovascular medicine, offering the opportunity to gather diagnostic information about the process of atherosclerosis in vivo, and to directly observe the effects of various interventions on the plaque and arterial wall. This book aims to give a comprehensive overview of this rapidly evolving technique from basic principles and instrumentation to research and clinical applications with future perspectives.

\title{
How to reference
}

In order to correctly reference this scholarly work, feel free to copy and paste the following:

Dermot Phelan, Sajjad Matiullah and Faisal Sharif (2012). Clinical Applications of Intravascular Ultrasound, Intravascular Ultrasound, Dr. Yasuhiro Honda (Ed.), ISBN: 978-953-307-900-4, InTech, Available from: http://www.intechopen.com/books/intravascular-ultrasound/clinical-applications-of-intravascular-ultrasound

\section{INTECH}

open science | open minds

\section{InTech Europe}

University Campus STeP Ri Slavka Krautzeka 83/A 51000 Rijeka, Croatia Phone: +385 (51) 770447 Fax: +385 (51) 686166 www.intechopen.com

\section{InTech China}

Unit 405, Office Block, Hotel Equatorial Shanghai No.65, Yan An Road (West), Shanghai, 200040, China 中国上海市延安西路65号上海国际贵都大饭店办公楼 405 单元 Phone: $+86-21-62489820$

Fax: $+86-21-62489821$ 
(C) 2012 The Author(s). Licensee IntechOpen. This is an open access article distributed under the terms of the Creative Commons Attribution 3.0 License, which permits unrestricted use, distribution, and reproduction in any medium, provided the original work is properly cited. 